\title{
Pentaquark Searches With CLAS
}

\author{
David J. Tedeschi for the CLAS Collaboration
}

Department of Physics and Astronomy, University of South Carolina, Columbia, SC 29208, USA

\begin{abstract}
The production of a possible, exotic $S=1$ pentaquarks off protons and neutrons is being investigated in Hall B at Jefferson Lab. Three dedicated experiments have been carried out to search for pentaquark signals using the CLAS detector in conjunction with a photon beam incident on hydrogen and deuterium targets. The status of on-going analyses and preliminary results from a variety of channels are presented.
\end{abstract}

Keywords: Pentaquark

PACS: 25.20.Lj, 25.75.Dw

\section{INTRODUCTION}

Since the 1960's when Gell-Mann introduced quarks and Nambu introduced color, baryons and mesons have been considered as three-quark and quark-antiquark color singlet configurations. This classification of known particles is largely unchanged today, yet searches for exotic configurations continue to play an important role in testing our understanding of the hadron spectrum. ${ }^{1}$ Exotic searches have gained new urgency based on experimental evidence that supported recent theoretical predictions for a new baryon state: the $\Theta^{+}(1540){ }^{2,3,4}$

This $S=1$ "pentaquark" baryon, composed of 5 quarks ( $u u d d \bar{s}$ ), was initially predicted to have a mass of $1540 \mathrm{MeV}$ and a width of $15 \mathrm{MeV}$. The state is exotic due to its strangeness and enigmatic due to its predicted narrow width. An early discussion by $\mathrm{Gao}$ and $\mathrm{Ma}$ of pentaquark search possibilities at Jefferson Lab generated little activity. ${ }^{5}$ However, since the announcement at PANIC 2002 by the LEPS group for a pentaquark signal in photoproduction data, ${ }^{6}$ there has been a renewed effort by the medium-energy and high-energy communities to analyze data for signals of exotic baryons.

At the present time there are both positive and negative results reported in the literature. ${ }^{7,8}$ Over the past year many of the new results reported have been negative, casting doubt on the existence of the pentaquark, but most of the new results are in different production channels and kinematic regimes than the positive signals. In response to the unclear experimental situation, the Hall B group has performed three experiments with the CLAS detector optimized to search for exotic particles. One experiment on deuterium (g10) and another on hydrogen (g11) improve the signal statistics for the $\Theta^{+}(1540)$ by an order of magnitude. Additionally, an effort is underway to search for exotic cascade particles (eg3). This endeavor aims to verify and search for further members of the pentaquark family of which the $\Theta^{+}(1540)$ is the lowest mass "anti-decuplet" state. These high-statistics experiments will provide 
reliable cross section limits that can be used to address the question of the existence of the exotic pentaquark states in a quantitative way.

\section{PHOTOPRODUCTION OF $\Theta^{+}(1540)$ ON DEUTERIUM}

Data published in 2003 provided the first claim from CLAS for the existence of an exotic $S=1$ baryon. ${ }^{9}$ A peak in the invariant mass $m\left(n K^{+}\right)$spectrum at $1540 \mathrm{MeV}$ with a width consistent with the experimental resolution was reported. The statistical significance of the peak was estimated at $5.8 \sigma$ when compared with a smooth background, and $4.8 \sigma$ when compared with a background shape determined from a Monte Carlo simulation. Preliminary results from the new experiment on deuterium (g10) that reproduce the conditions of our published $\Theta^{+}(1540)$ claim are shown in Figure 1. The missing mass of the $\mathrm{pK}^{-}$system for the reaction $\gamma+d \rightarrow p+K^{+}+K^{-}+(n)$ is presented where the black points are the older data published in Reference 9 and the red histogram is the new high-statistics data from CLAS scaled by 0.169 . No evidence for a peak at $1.54 \mathrm{GeV} / \mathrm{c}^{2}$ is seen in the new data. In addition to the order of magnitude increase in statistics, a monumental effort was put into understanding the CLAS calibration and detector corrections. From this data, CLAS has deduced a preliminary upper limit of 4-5 nb on the cross section of the $\Theta^{+}(1540)$ in this production channel. This result is undergoing final review in preparation for publication.

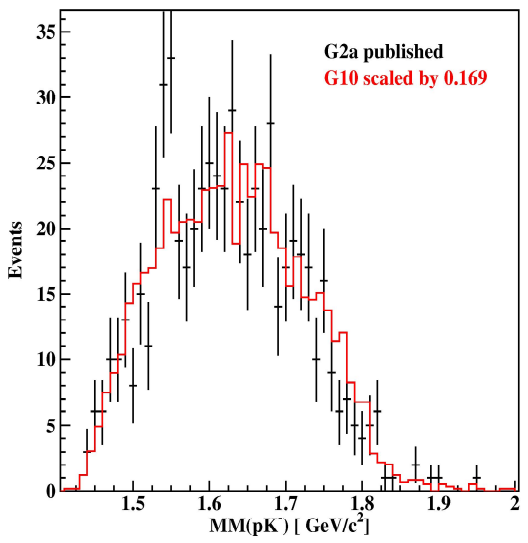

FIGURE 1. Preliminary missing mass of the $\mathrm{pK}^{-}$system for the reaction $\gamma+d \rightarrow p+K^{+}+K+(n)$. The black points are the data published in Reference 9 and the red histogram is the new high-statistics data from CLAS scaled by 0.169 .

Searches for the $\Theta^{+}(1540)$ in complementary channels are also underway using the CLAS g10 data. The high statistics makes possible for the first time the study of the channels $\gamma+d \rightarrow \Lambda+\Theta^{+}$and $\gamma+d \rightarrow \Lambda^{*}(1520)+\Theta^{+}$. The advantage of these channels is that they do not contain effects from hadronic interferences. On the other hand, understanding the role of the hadronic background is integral to clarify why so many 
laboratories reported a positive signal. The channel $\gamma+d \rightarrow p+K^{0}+K^{-}+(p)$ allows a check for exotic pentaquark production on the (quasi-free) neutron by detecting the decay mode $\Theta^{+} \rightarrow p+K^{0}$ through analysis of the $m\left(\mathrm{pK}^{0}\right)$ invariant mass distribution. The advantage of this channel is that no re-scattering mechanism is needed to produce a pentaquark and detection of the $\mathrm{K}^{-}$provides a strangeness tag for the $\mathrm{K}^{0}$. A study of interference effects from meson channels (such as the $\mathrm{a}_{2}(1320)$ ) that decay to $K^{0} K$ is underway, as these effects have been suggested as the cause of the structure in the mass region of $1.54 \mathrm{GeV} / \mathrm{c}^{2}$.

\section{PHOTOPRODUCTION OF $\Theta^{+}(1540)$ ON HYDROGEN}

The CLAS g11 run employed photons of energies in the interval $1.6 \mathrm{GeV}<E_{\gamma}<3.8 \mathrm{GeV}$ incident upon a $40 \mathrm{~cm}$ hydrogen target. Over seven billion triggers corresponding to an integrated luminosity of $70 \mathrm{pb}^{-1}$ were recorded. Each trigger required the detection of at least two charged particles in CLAS to facilitate the pentaquark search in the channels $\gamma+p \rightarrow K^{+}+K^{0}+(n)$ and $\gamma+p \rightarrow K^{0}+p+\left(K^{0}\right)$. In the former reaction, the intermediate state $\Theta^{+}(1540)$ decays to $\mathrm{nK}^{+}$while the latter measures the decay to $\mathrm{pK}^{0}$. The comparison of these channels, as well as the production data off the neutron will provide an important test of the $\Theta^{+}(1540){ }^{11}$ The analysis of $\gamma+p \rightarrow K^{0}+p+\left(K^{0}\right)$ is underway and the analysis of $\gamma+p \rightarrow K^{+}+K^{0}+(n)$ is described below.

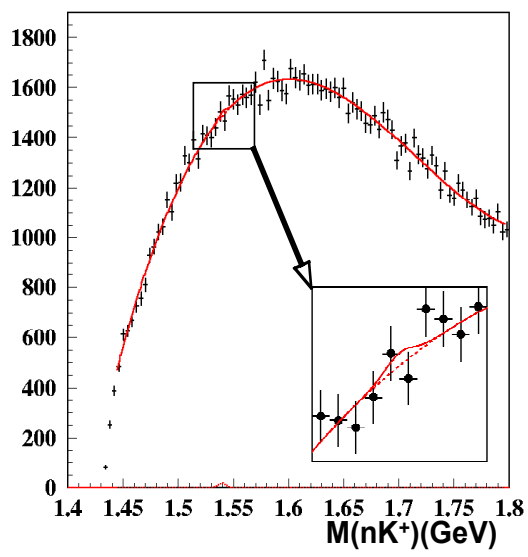

FIGURE 2. Invariant mass of the $\mathrm{nK}^{+}$system for the reaction $\gamma+p \rightarrow K^{+}+K^{0}+(n)$. Data are from the analysis of CLAS g11 data: $1.6 \mathrm{GeV}<\mathrm{E}_{\gamma}<3.8 \mathrm{GeV}$. Vertical axis is arbitrary yield.

The CLAS search for $\Theta^{+}(1540)$ production from hydrogen in the reaction $\gamma+p \rightarrow K^{+}+K^{0}+(n)$ has been completed and was first shown at the Spring APS (2005) meeting in Tampa, Florida. The analysis of this channel is a high statistics reproduction of the positive result published by the SAPHIR collaboration. ${ }^{12}$ The results of the CLAS measurement are shown in Figure 2. The invariant mass of the $\mathrm{nK}^{+}$system is smooth and shows no enhancement in the region of $1.54 \mathrm{GeV} / \mathrm{c}^{2}$. The 
CLAS data put an upper limit of $2 \mathrm{nb}$ on the production cross section in this channel. This number should be contrasted with the limit of $50 \mathrm{nb}$ from SAPHIR after a reanalysis of their published result of $300 \mathrm{nb} .^{12}$ It should also be noted that this result does not impact the previously published analysis on hydrogen by CLAS that is an analysis of $\Theta^{+}(1540)$ in the channel $\gamma+p \rightarrow K^{+}+K^{+}+\pi^{+}+(n)$ at photon energies $3 \mathrm{GeV}<E_{\gamma}<5.4 \mathrm{GeV} .{ }^{13}$ It is expected that data to verify this remaining CLAS result will be acquired in 2006/2007, depending on the Jefferson Lab run schedule.

\section{SEARCH FOR CASCADE PENTAQUARKS}

The CLAS Collaboration is involved in the search for the $\Phi^{--}$pentaquark state, another member of the exotic anti-decuplet. The eg3 run, successfully completed in early 2005 , consisted of a tagged photon beam of energy $4.5<\mathrm{E}_{\gamma}<5.5 \mathrm{GeV}$ incident on a $40 \mathrm{~cm}$ long deuterium target. A total of $100 \mathrm{pb}^{-1}$ of data - consisting of 4 billion two and three track triggers - was acquired during the run. A major part of the calibration, namely the timing calibration of tagger, start counter, time-of-flight detector, forward calorimeter, single-photoelectron calibration and the timing calibration for the Cerenkov Counter and RF-signal has been performed. At the present time, the calibrations have been finished.

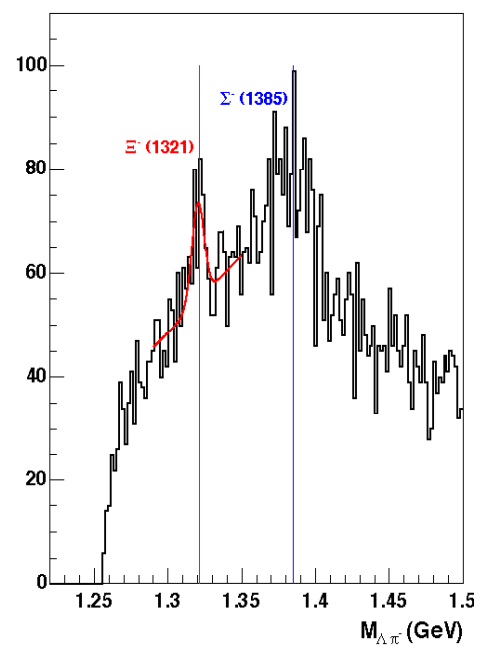

FIGURE 3. Reconstructed $\Lambda \pi^{-}$mass from $4 \%$ of eg 3 data. The lines indicate the mass of the $\Xi(1321)$ and the $\Sigma(1385)$. The fit is a gaussian with a polynomial background to estimate particle yield. Vertical axis is counts.

As a first step in the physical analysis, $p \pi^{+} \pi$ events have been reconstructed. The goal of extracting a well-known and kinematically over determined channel was to test detector systems and reconstruction code for their efficiency and to monitor this efficiency over the whole run period. In the case of the proton target it could be shown 
that the $\pi^{+} \pi^{-}$production channel can be isolated even in the absence of the magnetic field. This finding will be useful for future alignment routines for the drift chamber.

Another aspect of the physical analysis is the reconstruction of the $\Xi^{-}(1321)$ state from its decay products $\mathrm{p} \pi^{-} \pi^{-}$. The exotic $\Phi^{--}$is expected to decay via three subsequent interactions $\Phi^{--} \rightarrow \Xi^{-} \pi^{-}, \quad \Xi^{-} \rightarrow \Lambda \pi^{-}$, and $\Lambda \rightarrow p \pi^{-}$. The typical vertex displacement $(d=\gamma \beta \tau c)$ of $20 \mathrm{~cm}$ allows the reconstruction of intermediate decay vertices in regions with low physical background. This approach improves the signal to background ratio. Additionally, cutting on the invariant mass of the reconstructed $\Lambda$ 's reduces the background under the $\Sigma(1385)$ peak in the $m\left(\Lambda \pi^{-}\right)$invariant mass histogram (see Figure 3). We continue to develop this new and important reconstruction algorithm for CLAS to search for the $\Phi^{--}$. Starting with exclusive $p \pi \pi$ events from $4 \%$ of the data, the invariant $\Xi^{-}(1321)$ mass peak in Figure 3 becomes visible after applying narrow cuts on the $\Lambda$ mass and decay vertex and after demanding that the missing mass of the system is above the two-kaon threshold. The yield from this preliminary analysis is $\sim 100 \Xi^{-}(1321)$ 's which translates to $\sim 2500$ total $\Xi^{-}(1321)$ 's in the sample. This number is comparable to the yield from high-energy searches and will be used as a benchmark for comparison of the CLAS result to the world data. $^{14}$

\section{ACKNOWLEDGMENTS}

These measurements were made possible by the outstanding efforts of the staff of the Accelerator and the Physics Divisions at Jefferson Lab. This work was supported in part by the National Science Foundation under the award NSF-0244982. The Southeastern Universities Research Association (SURA) operates the Thomas Jefferson National Accelerator Facility for the United States Department of Energy under contract DEAC05-84ER40150.

\section{REFERENCES}

1. S. Eidelman et al., Phys. Lett. B 592, 1 (2004).

2. D. Diakonov, V. Petrov, M. Polyakov, Z. Phys. A 359, 305 (1997).

3. M. Karliner and H. Lipkin, Phys. Lett. B 575, 249 (2003).

4. R. Jaffe and F. Wilczek, Phys. Rev. Lett. 91, 232003 (2003).

5. H. Gao and B.-Q. Ma, Mod. Phys. Lett. A 14, 2313 (1999).

6. T. Nakano et al., Nucl. Phys. A 721, C112 (2003).

7. A. Dzierba, C. Meyer, A. Szczepaniak, J. Phys. Conf. Ser. 9, 192 (2005); hep-ex/0412077; A. Dzeirba et al., Phys. Rev. D 71, 098502 (2005).

8. K. Hicks, Prog. Part. Nucl. Phys. 55, 647 (2005); hep-ex/0504027.

9. S. Stepanayn, et al. (The CLAS Collaboration) Phys. Rev. Lett. 91, 252001 (2003).

10. Dzierba et al., Phys. Rev. D 69, 051901 (2004).

11. M. Karliner and H. Lipkin, hep-ph/0506084.

12. J. Barth et al., Phys. Lett. B 572127 (2003).

13. V. Kubarovsky et al. (The CLAS Collaboration) Phys. Rev. Lett. 92, 032001 (2004).

14. E.S. Ageev et al., hep-ex/0503033 (March 2005). 